\title{
El tránsito a la vida adulta de los jóvenes en dificultad social
}

\section{Miguel Melendro Estefanía}

Facultad de Educación

Universidad Nacional de Educación a Distancia (UNED)

<miguel.melendro@edu.uned.es>

Artikulu honetan, gizarte-zailtasun egoeran dauden gazteek helduarorako bidean, berriki hezkuntza eta gizarte-mailako esku-hartzeen inguruan egindako azterlan baten emaitzak azaltzen ditu. Gainera, berrikusi egin dira prozesu horretako ezaugarri nabarienak, eta profesionalek eta enpresariek gaiaren inguruan izandako jarrera-hartzeak aztertu. Gizarteratzean eta langintza-mailan izandako maila egokia nabarmentzen dute, eta balioeste bikaina egiten dute gazteek partaide izandako hezkuntza zein gizarteratzeko laguntzarako taldeen eta programen inguruan galdegitean. Enpresariek gogobetetzemaila jasoa erakusten dute programa hauekiko; aitzitik, nabarmentzen dute errendimendu baxua dutela gazte horiek. Hezkuntza eta gizarteratzeko taldeen esanaren arabera, baliabide urriak dituzte gazte horiek helduarorako bidean lagundu ahal izateko, eta gazte horien langintza eta erabateko gizarteratzeko aukera urriak aurreikusten dituzte.

\section{HITZ-GAKOAK:}

gazteak, gizarte-zailtasuna, hezkuntza eta gizartemailako esku-hartzea, emantzipazio, gizarte iraunkor.
En este artículo se dan a conocer los resultados de una reciente investigación sobre los efectos de la intervención socioeducativa en el tránsito de los jóvenes en dificultad social a la vida adulta. Se revisan además las características más destacadas de ese proceso, junto a los posicionamientos de profesionales y empresarios ante él. Los resultados muestran el aceptable nivel de inserción sociolaboral alcanzado por los jóvenes, además de su excelente valoración de los equipos socioeducativos y los programas en los que han participado. Los empresarios expresan un elevado grado de satisfacción con estos programas, aunque opinan que el rendimiento laboral de los jóvenes es bajo. Los equipos socioeducativos, por su parte, señalan la escasez de recursos para apoyar el tránsito de los jóvenes a la vida adulta y muestran bajas expectativas sobre su inserción sociolaboral final.

\section{Palabras clave:}

jóvenes, dificultad social, intervención socioeducativa, emancipación, sociedad sostenible. 


\section{Contexto y motivaciones de la investigación}

En los últimos años, parece despertar cada vez más el interés de los expertos e investigadores por los modelos de intervención socioeducativa con adolescentes y jóvenes en dificultad o en desventaja social, especialmente cuando se trata de jóvenes que se encuentran en el periodo de la denominada adolescencia tardía, entre los 16 y los 19 años de edad. Se trata de jóvenes que se enfrentan, más tempranamente que otros de su generación, al reto de iniciar una vida adulta autónoma e independiente en el momento mismo en que cumplen los 18 años y alcanzan la mayoría de edad.

Algunas de las aportaciones más interesantes y actuales en este ámbito de trabajo e investigación (Du Bois y López Blasco, 2004; Bendit y Stokes, 2004; Inglés, 2005) vienen a señalar que estos 'adultos jóvenes' se encuentran en un momento especialmente vulnerable de su trayectoria vital. Un momento de necesidad imperiosa de apoyos externos significativos y continuados que les ayuden a realizar esa transición de la vida adolescente a la vida adulta con ciertas garantías de éxito. Se plantea, en ese sentido, la necesidad de profundizar en el apoyo institucional a adolescentes y jóvenes en dificultad social, a través de nuevas 'políticas de transición', unas políticas sociales que han de tener en cuenta tanto las particulares características y necesidades de esta población como sus circunstancias generacionales, caracterizadas por cambios frecuentes y recursivos en su vida cotidiana: en el lugar donde residen, en los espacios que frecuentan, en el trabajo, en las relaciones de pareja, personales y familiares o en la ocupación de su tiempo de ocio.

Siendo relativamente fácil encontrar investigaciones de tipo descriptivo que abordan de forma genérica esta temática, la mayoría de ellas relacionadas con el campo de la sociología (Walther y Pohl, 2007; Inglés et al., 2005; Du Bois-Reymond y López, 2004; Rahona y Vaquero, 2003), no se cuenta, sin embargo, con estudios de cierta relevancia que aborden el tránsito a la vida adulta de los jóvenes en dificultad o desventaja social, y aporten información y propuestas de trabajo contrastadas sobre la intervención socioeducativa que se realiza con ellos.

Partiendo de esta situación, decidimos llevar a cabo este estudio a partir de la experiencia de trabajo de más de diez años con más de tres mil adolescentes y jóvenes en dificultad social, desde diferentes programas de intervención en la Comunidad de Madrid $^{1}$. Nos parecía de sumo interés profundizar en

${ }^{1}$ Esta investigación se ha llevado a cabo gracias a la financiación y el apoyo recibido por parte de Opción3, S. Coop., y la Universidad Nacional de Educación a Distancia (UNED). Opción 3, las características, los fundamentos y los resultados obtenidos con las metodologías y estrategias de intervención socioeducativa utilizadas con esta población, y muy especialmente con aquellas estrategias que, desde modelos innovadores y con claros efectos multiplicadores, procuran un mayor éxito en la inserción personal, social y laboral de estos jóvenes en dificultad social.

Tomamos en consideración también que la propia Unión Europea había introducido ya en su agenda la atención prioritaria a este colectivo, iniciando una línea de trabajo sobre la inclusión social de jóvenes desfavorecidos. El informe de la Comisión Europea sobre inclusión social, publicado en mayo de 2004 , incorporaba a los jóvenes desfavorecidos como un grupo objetivo estratégico, y planteaba abordar estas situaciones de desventaja desde dos ámbitos prioritarios: la educación y formación de los jóvenes, y el incremento de su participación en el mercado laboral. El Pacto Europeo de la Juventud (2005), que forma parte de la Estrategia de Lisboa, establecía, por su parte, "como una medida imprescindible para el crecimiento sostenible e inclusivo de Europa la integración social de los jóvenes”. Estas iniciativas siguen el camino iniciado por el libro blanco Un nuevo impulso para la juventud europea (2001), y a esta prioridad europea se suman los trabajos desarrollados en la presente investigación, que indaga sobre la situación de los jóvenes desfavorecidos en nuestro país, y sobre las estrategias socioeducativas más adecuadas para su tránsito a la vida adulta y su inclusión en una sociedad sostenible.

Lorenzo Cachón (2004) aporta cuatro elementos que inciden en estos nuevos procesos de transición de los jóvenes a la vida adulta, aspectos que considera comunes a la mayoría de los países de la Unión Europea. Para este autor:

- Se ha alargado de forma considerable la formación inicial de los jóvenes, especialmente en países como Italia, Reino Unido o España.

- Se ha producido una importante transformación de la estructura del empleo y de la naturaleza de las cualificaciones requeridas a los jóvenes, debido a los cambios y las innovaciones en los ámbitos tecnológicos y de organización del trabajo.

S. Coop., es una entidad que desarrolla diferentes programas de intervención socioeducativa con adolescentes y jóvenes en dificultad social de la Comunidad de Madrid (programas Junco, Elfos y Alcor, dirigidos a menores tutelados, menores con medidas judiciales, menores extranjeros no acompañados y población adolescente y juvenil en riesgo de exclusión social), que se han desarrollado en colaboración con instituciones públicas como el Instituto Madrileño del Menor y la Familia, la Concejalía de Gobierno de Empleo y Servi cios al Ciudadano del Ayuntamiento de Madrid, y la Agencia de la Comunidad de Madrid para la Reeducación y Reinserción del Menor Infractor, entre otras. 
- Se ha endurecido la selección de la mano de obra por parte de las empresas, en relación directa al incremento de la competitividad en el mercado.

- Se han institucionalizado nuevos espacios de formación y empleo, a partir de la intervención de los poderes públicos para establecer mecanismos especiales de inserción laboral de los jóvenes.

Efectivamente, el retraso en la incorporación al trabajo protagonizado en gran medida por los jóvenes estudiantes de clase alta y media ha hecho que hablar de jóvenes activos entre los 16 y 19 años sea hablar de jóvenes de origen social medio-bajo o bajo. Un grupo que ha optado, junto a mujeres e inmigrantes, por las ramas de actividad laboral menos codiciadas (Cachón, 1998). Se trata de jóvenes vulnerables, que tienen menos recursos personales y sociales, y que no disponen de demasiado tiempo para efectuar con garantías de éxito su tránsito a la vida adulta, ya que las instituciones públicas se retiran cuando estos jóvenes cumplen la mayoría de edad.

En nuestro país, un fenómeno destacable, junto a los planteados por Cachón (2004), es la elevada y preocupante tasa de abandono y fracaso escolar. La escolarización, en el contexto europeo, responde al criterio -también sustentado por Naciones Unidas- de que, además del acceso a la enseñanza, hay que lograr que ningún niño abandone la escuela y que todos reciban una educación de calidad. Los objetivos de la Unión Europea, en este sentido, son incrementar, en 2010 , el porcentaje de personas que han terminado la Educación Secundaria Postobligatoria, pasando del 74\% de 2009 -el $63,4 \%$ en España- al $85 \%$; y reducir, en el mismo periodo, el abandono escolar prematuro en Bachillerato y Formación Profesional por debajo del $10 \%$. Mientras que la media europea de abandono escolar es de un $14,8 \%$, y países como Alemania, Bélgica, Francia o Reino Unido rondan el $12 \%$, nuestro país no sólo tiene un porcentaje muy elevado, sino que además lo ha incrementado en 1,1 puntos en 2009, llegando al 31\%, es decir, el doble prácticamente que la media europea, y sólo por encima de Portugal (36,3\%) [López Blasco, 2007; Ministerio de Educación, 2008]. A esto se añade el dato de que los estudiantes españoles de 15 años -Secundaria Obligatoria- están por debajo de la media de los países desarrollados (OCDE) en nivel de lectura, matemáticas y conocimiento científico, según el Informe del Programme for International Student Assessment (PISA, 2006).

A esta situación, hemos de añadir los efectos de la grave crisis por la que atraviesa el sistema económico mundial. Una crisis compleja e incierta, sin duda duradera, por lo que supone de cambio global. Como ya se está comprobando, los sectores más vulnerables son quienes finalmente han sufrido mayoritariamente las repercusiones de esta crisis económica global. Inmigrantes, jóvenes no cualificados, mujeres con o sin cargas familiares, trabajadores ahora parados del mundo de la construcción o del automóvil... se enfrentan a un mercado cada vez más restringido y restrictivo, más exigente con las competencias, con la formación, con el esfuerzo requerido y con los salarios de sus trabajadores.

En nuestro país, por ejemplo, muchos jóvenes que dejaron las aulas en busca de un empleo fácil en el sector turístico o en el de la construcción, se encuentran hoy sin título y sin trabajo. Por miedo al paro, o a perder oportunidades, los jóvenes adultos están volviendo a clase, en busca del graduado escolar, el título de formación profesional o incluso el universitario. En la actual situación de crisis económica, en la que las posibilidades de encontrar un empleo de baja cualificación son cada vez menores, las administraciones esperan que las matriculaciones en los nuevos programas de formación profesional se multipliquen. En respuesta a esta coyuntura, una serie de propuestas del Ministerio de Educación para la nueva Formación Profesional plantean flexibilizar y multiplicar las alternativas al sistema educativo ordinario para reenganchar a los jóvenes excluidos, en línea con la filosofía de políticas integrales de juventud anteriormente comentada.

Los profesionales y las entidades que trabajan con esta población se encuentran también con problemas más difíciles de resolver, con personas con menos tiempo para encontrar un empleo, con menos ofertas de empleo y más exigentes. Lo que supone para ellos más esfuerzo, más formación, más creatividad e imaginación. Se requerirán nuevos espacios para poder analizar, sopesar, tomar decisiones compartidas, acertadas; y también más flexibilidad para abordar lo incierto, lo impredecible, lo precario... Algo que ya forma parte del 'currículum' de los profesionales de lo social, pero sobre lo que deberán ejercitarse y formarse para actuar de una forma más fundamentada y eficaz.

\section{El estado de la cuestión: otros estudios de referencia}

Como señalábamos anteriormente, la juventud en dificultad social y su tránsito a la vida adulta en la sociedad actual ha sido objeto de numerosas investigaciones en los últimos años. Estas investigaciones, de alcance internacional y marcado carácter sociológico, pretenden conocer en profundidad la situación de los jóvenes desfavorecidos y las causas de su exclusión social. Se pueden mencionar, al respecto, tres líneas de investigación bien desarrolladas: 
- Una primera que incide en la caracterización del periodo socioevolutivo de la adolescencia tardía y su identificación -en parte- con el grupo de población actualmente denominado de 'jóvenes adultos', y con el momento del tránsito a la vida adulta.

- En segundo lugar, otro grupo de investigaciones que tratan sobre las 'trayectorias fallidas' a que son abocados los jóvenes más vulnerables buscan su descripción detallada, y plantean la necesidad de reestructurar las políticas sociales de atención a este colectivo.

- Por último, un grupo de trabajos, menos pródigo, que recoge información sobre 'buenas prácticas' en la intervención socioeducativa con jóvenes adultos en dificultad social; experiencias y propuestas que responden al objetivo último de lograr una sociedad más solidaria y sostenible.

Respecto a la primera línea de trabajo, ya mencionábamos cómo las recientes investigaciones de autores como Du Bois-Reymond y López Blasco (2004), Bendit y Stokes (2004), Machado País y Pohl (2004) o Cachón (2004) no sólo caracterizan la realidad de los 'jóvenes adultos', sino que también señalan cómo éstos se encuentran en un momento especialmente vulnerable de su trayectoria vital y necesitados de apoyos externos importantes para poder realizar esa transición, temprana y obligada, de la vida adolescente a la vida adulta con ciertas garantías de éxito.

La segunda línea de trabajo profundiza en el reconocimiento de las 'trayectorias fallidas' de los jóvenes más vulnerables. En nuestro país, diversas investigaciones han indagado longitudinalmente sobre la situación adulta de quienes fueron menores protegidos (Fernández del Valle, 1998; Inglés, 2005; García Barriocanal, Imaña y De la Herrán, 2007; Casas y Monserrat, 2009, Melendro, 2010). Estas investigaciones relacionan aspectos contextuales de tipo institucional, familiar o socioeconómico de la historia personal de los menores, con una serie de descriptores básicos sobre su situación adulta, el mayor o menor grado de inserción social logrado y, en alguno de los casos, con las intervenciones socioeducativas desarrolladas. Así, desde el Proyecto Telémaco (Inglés, 2005), centrado en las buenas prácticas de los procesos de socialización de adolescentes y jóvenes tutelados que han alcanzado la mayoría de edad, o están próximos a alcanzarla, se señala cómo "es del todo contraproducente interrumpir la intervención educativa y socializadora al cumplir [el joven] la mayoría de edad" (Inglés, 2005), aspecto claramente relacionado con la importancia de evitar 'políticas lineales', que priman los aspectos administrativos sobre las necesidades y posibilidades reales de integración social de esta población. Otras investigaciones en España han indagado longitudinal- mente sobre la situación adulta de quienes fueron menores protegidos (Fernández del Valle, 1998; García Barriocanal, Imaña y De la Herrán, 2007), relacionando aspectos contextuales de tipo institucional, familiar o socioeconómico de la historia personal de los menores con una serie de descriptores básicos sobre su situación adulta, y su mayor o menor grado de inserción social.

Por otra parte, diversos estudios norteamericanos, tanto canadienses (Yergueau, Pauzé y Toupin, 2007; Goyette et al., 2004, 2007) como estadounidenses (Lenz-Rashid, 2006; Stein, 2006; Reilly, 2003) abordan el tema del tránsito a la vida adulta de los jóvenes en dificultad social, centrados tanto en factores descriptivos del contexto y de las diferentes situaciones de riesgo por las que atraviesan los jóvenes, como en las características e incidencia de la intervención socioeducativa con ellos.

En relación a la tercera línea de investigación mencionada, que hace referencia a las buenas prácticas en la intervención socioeducativa, Yergeau, Pauzé y Toupin (2007) concluyen recomendando que los programas de intervención con estos jóvenes adultos vayan más allá de la mejora de la empleabilidad, e incorporen apoyos emocionales antes y durante la transición, apoyo para el establecimiento de relaciones afectivas y sociales positivas más allá de las familiares, así como intervenciones complementarias centradas en la reducción del consumo de sustancias tóxicas y del estrés ligado a la autonomía precoz a la que se ven forzados.

En este tercer grupo de investigaciones, podemos situar también el trabajo dirigido por Martin Goyette (2007), que avanza en el estudio de estrategias de intervención a través de modelos socioeducativos novedosos, como el Projet Qualifications des Jeunes $(P Q J)$, o en el diseño de instrumentos de diagnóstico sobre el tránsito a la vida adulta construidos participativamente con jóvenes procedentes del sistema de protección canadiense, como la Evaluation du Niveau de l'Autonomie (EVA). Algunas conclusiones relevantes señalan cómo la intervención debe reposar sobre la construcción de una relación fuerte del educador con el joven, de forma que el interviniente se convierta en una persona significativa para él o para ella; se destaca también la importancia de saber construir desde la cohabitación de los paradigmas de intervención, a través del trabajo en red e interdisciplinar. Se defiende, a su vez, la necesidad de sostener procesos de adquisición de competencias y de experiencias sociales esenciales para la transición a la vida adulta, así como de intervenir de forma 'sostenible' -duradera en el tiempo y con una cierta intensidad $-\mathrm{y}$ flexible. Todo ello con dos prerrequisitos imprescindibles: la disponibilidad de empleos de la mayor calidad posible, y de alojamiento asequible y adecuado. 
Por nuestra parte, después de revisar el estado de las investigaciones y las aportaciones teóricas en este campo de trabajo, nos planteamos, con la investigación descrita en este artículo, avanzar en el conocimiento de las características y los efectos de una metodología que se basa en el desarrollo de 'estrategias flexibles' de intervención socioeducativa. Esta metodología se centra en la elaboración de planes de acción ecosociales, que requieren un conocimiento preciso del escenario relacional de los jóvenes, y que atienden tanto a las posibilidades reales de intervención como a la carga de incertidumbre que acompaña a las situaciones abordadas ${ }^{2}$.

\section{Diseño, metodología y limitaciones de la investigación}

La investigación que a continuación se describe se desarrolló entre 2005 y 2008 , a través de una batería de instrumentos aplicados a diferentes grupos de población -jóvenes, empresarios y educadores-, con la finalidad de profundizar en el conocimiento de la situación de los jóvenes en dificultad social, una vez han transitado a la vida adulta y se han distanciado de los dispositivos de protección social por los que fueron atendidos en su infancia y adolescencia.

Nos planteamos, a partir de esta finalidad, un grupo de objetivos acordes a la compleja realidad abordada y a los efectos que la intervención socioeducativa produce en ella (Figura 1).

Figura 1. Diseño de la investigación

SISTEMA SOCIO-ECONÓMICO

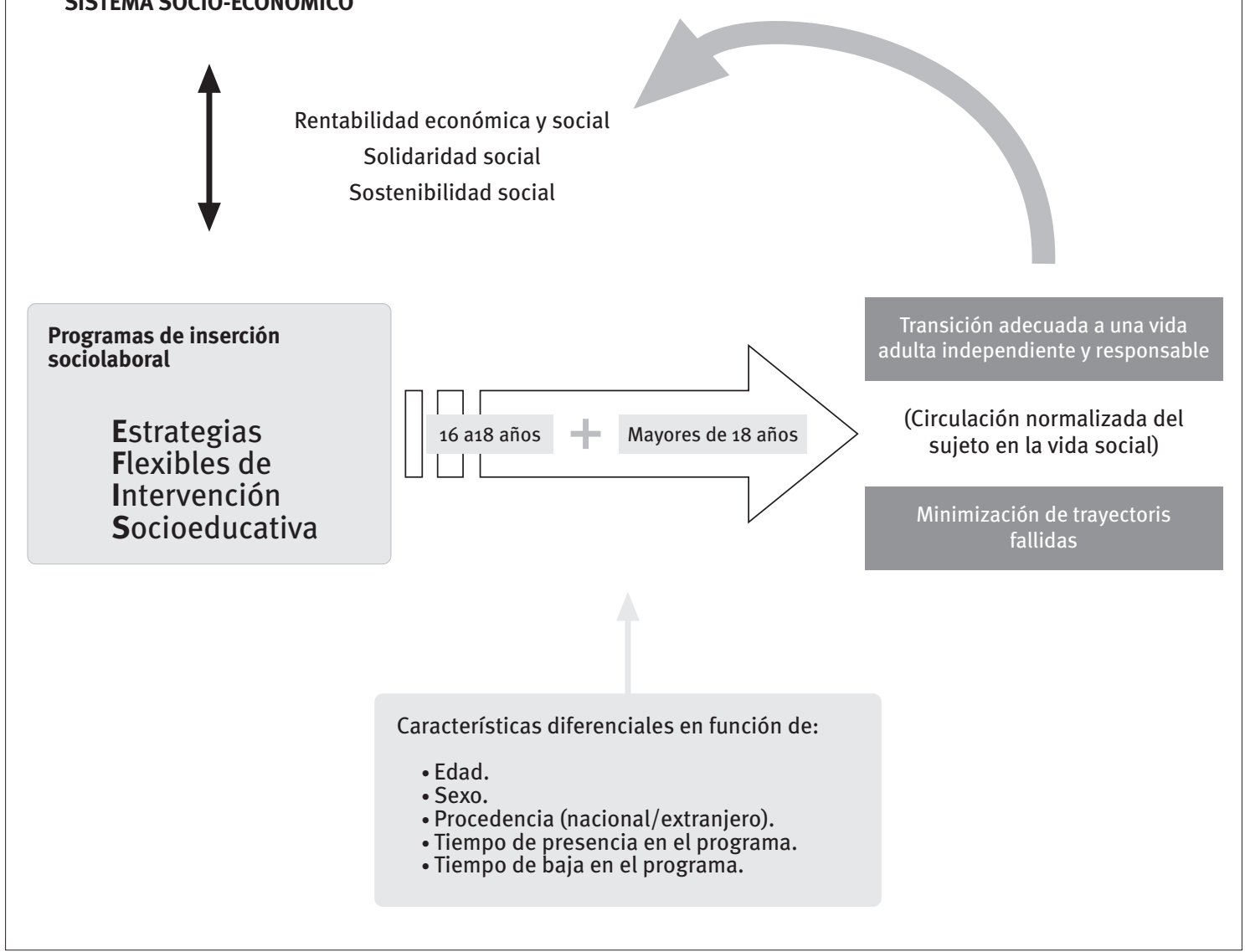

Fuente: Elaboración propia. 
Desde este planteamiento, se llevó a cabo una investigación ex post facto de tipo causal-correlacional, que analizaba longitudinalmente la información disponible en torno al momento de la emancipación e incorporación a la vida adulta autónoma de los jóvenes. La metodología utilizada combina el análisis de datos paramétricos de tipo descriptivo e inferencial -a través de técnicas de análisis bivariable y multivariable- $y$ de tipo cualitativo.

Los sujetos centrales de estudio son los adolescentes y jóvenes en dificultad social con los que se han realizado intervenciones socioeducativas desde programas de inserción sociolaboral, a edades comprendidas entre los 16 y los 19 años. La población investigada es de 1.268 sujetos, población de la que se procedió a extraer una muestra aleatoria que, para un nivel de confianza del $99 \%$ y un margen de error de 0,1 , fue de 87 sujetos.

Otro grupo que forma parte de la investigación es el de los empresarios que proporcionan ofertas de trabajo a esta población. De 986 empresas colaboradoras con los programas de empleo en los últimos diez años, se descartaron para la investigación aquellas que realizaron un único contrato o que no mantuvieron contacto durante los últimos años con la población de jóvenes seleccionada. Se utilizó, así, como población un total de 118 empresas, sin proceder a ningún tipo de muestreo, con la expectativa de que la mayoría de ellas respondieran al cuestionario. Así ocurrió, pues 88 (75\%) lo cumplimentaron.

Un último grupo que participó en el estudio fue el de los educadores y profesionales que trabajaban en los programas de inserción sociolaboral con la población de jóvenes y empresarios seleccionada. En total, se encuestó a un total de 36 profesionales con entre uno y veinte años de experiencia en este ámbito de trabajo, y una media de seis.

Los instrumentos y técnicas de investigaciones utilizadas y los grupos de población a los que se han dirigido se pueden apreciar en la tabla 1.

Tanto el cuestionario a empleadores como el cuestionario a profesionales ofrecieron un elevado grado de consistencia interna (alfa de Cronbach de o,913 y de 0,859 , respectivamente). La entrevista y el cuestionario a jóvenes muestran un grado de sinceridad en la respuesta de entre bueno y muy bueno $(98,7 \%)$, a la vez que un elevado nivel de colaboración de los jóvenes en su realización (96,2\%).

Por último, conviene mencionar una serie de aspectos que limitan el alcance de este tipo de investigaciones. Por una parte, la población estudiada resulta especialmente difícil de localizar y de abordar, lo que se traduce en un esfuerzo importante a la hora de realizar en el trabajo de campo. Junto a ello, la utilización de instrumentos de recogida de datos autoperceptivos hace necesario introducir una serie de elementos de control: fiabilidad interjueces, triangulación de los valores obtenidos en las variables, descripción detallada del proceso y características del estudio que faciliten sus posteriores réplicas, y mejoren su fiabilidad y validez, así como la aplicación de otras medidas correctoras recomendadas por la literatura para este tipo de investigaciones y para las técnicas empleadas.

Tabla 1. Instrumentos y técnicas de investigación

\begin{tabular}{|c|c|}
\hline Instrumento & Principales características \\
\hline $\begin{array}{l}\text { - Estudio y análisis estadístico } \\
\text { de bases de datos. }\end{array}$ & $\begin{array}{l}\text { - Bases de datos de: } \\
\text { a. Población de adolescentes y jóvenes en dificultad social atendidos en programas de inserción } \\
\text { sociolaboral (1.268 sujetos). } \\
\text { b. Empresas/empleadores que han colaborado con los programas de inserción sociolaboral } \\
\quad \text { (986 empresas). } \\
\quad \text { - Se obtuvo información sobre la delimitación y caracterización de contexto de la investigación. }\end{array}$ \\
\hline $\begin{array}{l}\text { - Estudio de historias } \\
\text { profesionales (titulación, } \\
\text { experiencia y formación). }\end{array}$ & $\begin{array}{l}\text { - Estudio de historias de } 36 \text { profesionales del ámbito de la intervención socioeducativa con adolescentes y } \\
\text { jóvenes en dificultad social. } \\
\text { - Se obtuvo información sobre la biografía, la formación, la experiencia y el perfil profesional de los equipos } \\
\text { socioeducativos que trabajan con esta población. }\end{array}$ \\
\hline - Cuestionario a profesionales. & $\begin{array}{l}\text { - Cuestionario escrito cumplimentado por } 36 \text { profesionales. } \\
\text { - Se obtuvo información sobre los principios, los modelos, las estrategias y las expectativas de intervención } \\
\text { por parte de los profesionales. }\end{array}$ \\
\hline $\begin{array}{l}\text { - Entrevista semiestructurada } \\
\text { y cuestionario escrito a } \\
\text { jóvenes. }\end{array}$ & $\begin{array}{l}\text { - Entrevista y cuestionario aplicados a los } 87 \text { adolescentes y jóvenes en dificultad social de la muestra } \\
\text { establecida. } \\
\text { - Se obtuvo información sobre la situación actual de los jóvenes (laboral, familiar, social, personal), su trán- } \\
\text { sito a la vida adulta, los aprendizajes significativos atribuidos a la intervención socioeducativa y sus expec- } \\
\text { tativas y propuestas de futuro. }\end{array}$ \\
\hline - Cuestionario a empresarios. & $\begin{array}{l}\text { - Cuestionario escrito cumplimentado por } 88 \text { empresas/empleadores que colaboraban con los programas de } \\
\text { inserción sociolaboral. } \\
\text { - Se obtuvo información sobre la intervención socioeducativa realizada, propuestas de mejora sobre ésta y } \\
\text { sobre el trabajo de los jóvenes en las empresas. }\end{array}$ \\
\hline
\end{tabular}




\section{El escenario de la intervención}

Seguidamente se reflejan los resultados de la investigación y algunas de las interpretaciones y conclusiones más importantes, tomando como referencia los objetivos formulados inicialmente y que han guiado todo el proceso. El primer objetivo del estudio plantea delimitar y caracterizar el contexto en que se desarrolla la intervención socioeducativa para la inserción social y laboral de adolescentes y jóvenes en dificultad social. Los elementos más relevantes de esta descripción del contexto son:

- La población investigada es de 1.268 adolescentes y jóvenes procedentes de 141 centros diferentes del sistema de protección. Se trata de cifras muy relevantes, por lo que suponen en cuanto a la implicación de prácticamente toda la red constituida en torno a la intervención socioeducativa con adolescentes y jóvenes en dificultad social de la Comunidad de Madrid.

- La mitad de estos adolescentes y jóvenes han permanecido más de un año ingresados en un centro de menores; un 20\%, más de dos años; y un $9 \%$, más de tres.

- El porcentaje de población femenina atendida es de un $36 \%$, y cerca de una tercera parte (31\%) es población inmigrante, de más de treinta nacionalidades diferentes.

- Su formación inicial es muy precaria: proceden, sobre todo, de una Enseñanza Secundaria Obligatoria sin culminar, o de programas de diversificación o compensación educativa $(86,8 \%)$.

- Son atendidos principalmente desde las instituciones de protección y atención a la infancia, y los programas y servicios de inserción sociolaboral. Estos últimos desempeñan, a la vez, funciones de búsqueda directa y de mediación entre los empleadores, los jóvenes y el dispositivo de protección o atención social del que estos últimos proceden. Los equipos socioeducativos que los constituyen están formados por personas jóvenes -de en torno a los 30 años de edad-, en una proporción similar de hombres y mujeres, y con titulaciones de Psicología, Educación, Sociología o Derecho. Se trata de profesionales con un buen nivel formativo inicial, que además han recibido una amplia formación complementaria, centrada especialmente en la intervención socioeducativa con menores y en la planificación y gestión de recursos educativos. Son profesionales que además, en bastantes casos, han ocupado temporalmente, en su adolescencia y primera juventud, puestos de trabajo similares a los que ocuparán los jóvenes con los que van a trabajar en los programas de empleo.

- Las empresas que dan trabajo a los jóvenes son pequeñas, familiares o con pocos empleados, mientras que las medianas y grandes suponen menos de la mitad de las que colaboran con este tipo de programas. Comprobamos además cómo sólo un pequeño grupo de empresas $(3,1 \%)$ proporcionan un número elevado de ofertas de empleo y de contrataciones (Tabla 2).

- Los tipos de contrato predominantes son los temporales, de formación y por obra. Sólo un 3\% son de carácter indefinido. En general, el tiempo de contratación es reducido, siendo la mayoría de los contratos de menos de seis meses.

- La mayoría de los jóvenes trabajan en los sectores de hostelería, construcción, comercio y alimentación.

Tabla 2. Contratos por empresas

\begin{tabular}{l|c|c}
\hline № de contratos & Empresas & $\%$ \\
\hline 1 & 777 & $78,8 \%$ \\
\hline $2-5$ & 178 & $18,1 \%$ \\
\hline $6-20$ & 29 & $2,9 \%$ \\
\hline >20 & 2 & $0,2 \%$ \\
\hline Total & 986 & $100,0 \%$ \\
\hline
\end{tabular}

Fuente: Elaboración propia.

\section{El tránsito de los jóvenes a la vida adulta $^{3}$}

El segundo objetivo de la investigación plantea conocer las características del tránsito a la vida adulta efectuado por los jóvenes en dificultad social que participan en programas de inserción sociolaboral: su situación personal, social y económica tras la emancipación. Estamos hablando de una población que habita mayoritariamente en la periferia urbana y metropolitana de Madrid, y cuya presencia en el ámbito rural es prácticamente inexistente.

Son jóvenes que viven, en su gran mayoría, en pisos, propios o compartidos, con una gran movilidad, que cambian con cierta facilidad de lugar de residencia y también de núcleo de convivencia. Viven con la familia de origen, con amigos, con compañeros de piso o de trabajo, compartiendo el pago del alquiler. Algunos han constituido su propia familia; son tantos como los que viven solos, en ambos casos en torno al $10 \%$ de la población investigada. Según la información disponible, podemos considerar también que al menos un 1,2\% de los jóvenes atendidos por este tipo de programas acaban viviendo en la calle.

Es, en este sentido, importante constatar que la vivienda, el trabajo y el grupo primario de conviven-

3 La población juvenil investigada tiene edades comprendidas entre los 18 y los 26 años, siendo las edades más representadas las comprendidas entre los 19 y los 22 años (80\% del total). 
cia son elementos escasamente interrelacionados, entre los que no se pueden establecer asociaciones estadísticamente significativas, ni grupos definidos. Habría que considerarlos más bien como elementos de ese itinerario fluctuante e incierto que recorren los jóvenes en dificultad social.

La inmensa mayoría de estos jóvenes (90,7\%) afirman llevarse bien, o muy bien, con las personas con las que conviven; en general, tienen un mundo de relaciones amplio y fluido, muestran una adecuada socialización y una red de apoyo social bastante extensa. En casa, la mayoría hacen las tareas del hogar (cocinar, hacer la compra, hacer la limpieza, planchar) habitual o frecuentemente. Sus principales aficiones son, por este orden, hacer deporte, quedar con amigos o con la pareja, y salir de fiesta. Las actividades de tipo cultural (leer, ir al cine, escuchar música) ocupan sólo una pequeña parte de sus prioridades. El mundo asociativo no parece resultarles especialmente atractivo: sólo dicen estar asociados a algún tipo de entidad el 14,8\% de ellos. Es un nivel muy bajo de participación social, aunque se trata de una característica bastante común y conocida de los jóvenes de esta generación ${ }^{4}$. Por otro lado, entre el $65,5 \%$ y el $82,4 \%$ mantiene aún relación con personas que conocieron en los programas de inserción sociolaboral o en los centros de protección de menores, tanto educadores/as como antiguos/as compañeros/as, que, en algún caso, se han convertido en amigos cercanos, novios/as o compañeros de trabajo.

En el momento de ser entrevistados, dos tercios de los jóvenes se encontraban trabajando (64\%). La tasa de paro de los jóvenes en dificultad social es, por tanto, elevada, en comparación con la media europea en el momento de hacer la investigación $(16,8 \%)^{5}$, aunque dadas las especiales condiciones de acceso al empleo de estos jóvenes, un porcentaje de ocupación como el encontrado no es una mala noticia. En cuanto a los motivos para abandonar el trabajo en esta etapa de su vida, los jóvenes mencionan, sobre todo, circunstancias que hacen ver la difícil realidad laboral a la que, en ocasiones, deben enfrentarse ("era un trabajo muy duro", "no tenía contrato", "me pagaban en negro", "no pagaban bien", "tenía problemas médicos"). Otras respuestas ("me dio un venazo", "me rallé") ponen en evidencia las dificultades personales que tienen ellos mismos a la hora de enfrentarse a la búsqueda y, sobre todo, a la conservación del empleo. El salario medio de los jóvenes investigados es de 854 euros al mes. Prácticamente la mitad gana entre 500 y 900

4 Según el informe Jóvenes Españoles 2010 (González-Anleo, J.; y González Blasco, 2010), sólo el 19\% de los jóvenes manifiestan pertenecer a algún tipo de asociación.

5 Según datos publicados por Eurostat, en enero de 2007 el paro juvenil alcanzaba una medida del $16,8 \%$ en la UE-27, y llegaba al $20,4 \%$ en febrero de 2011. euros netos al mes. Es ésta, por tanto, una población que supera los ingresos del salario mínimo interprofesional, claramente aspirante a 'mileurista'.

A los jóvenes entrevistados les gusta lo que hacen en su trabajo: encontramos que es así para tres cuartas partes de ellos. La nota media con la que califican a sus trabajos es de 6,78 puntos sobre 10 , es decir, una calificación próxima al 'notable', con una desviación típica poco elevada $(1,72)$. Los jóvenes se encuentran, en términos generales, a gusto en su trabajo; consideran que lo desempeñan adecuadamente y valoran el buen ambiente existente en él. La inmensa mayoría expresa que el trabajo es muy importante para poder vivir como una persona adulta independiente y responsable. Afirman que el trabajo:

Te ayuda a ser responsable y a saber cuál es tu escala de valores en la vida; te crea una rutina diaria, te enseña a saber estar y el trato social... Personalmente, sin trabajo me siento muy mal; el no colaborar en casa económicamente o depender de mi madre, con los gastos que hay en casa, me hunde la moral... Las cosas no se pagan solas, y si no te apañas por ti mismo, la gente te ayuda un poco, pero tiene un límite y no se debe abusar...

Cuando se les pregunta si estaban mejor antes -como menores 'protegidos'- 0 ahora, la respuesta es muy clara: una mayoría abrumadora manifiesta encontrarse mejor en su situación actual ( $82 \%)$. Ahora se sienten más independientes y responsables de sus vidas, más libres para tomar sus propias decisiones. Aunque lo más duro en el tránsito fue vivir y salir adelante en soledad: sumando la falta de apoyos externos a esa responsabilidad de tener que 'salir adelante solo/a', nos encontramos con la realidad de la mitad de estos jóvenes. La inestabilidad laboral y los problemas con la vivienda completan un panorama poco deseable.

Casi tres de cada cuatro jóvenes (73,3\%) entrevistados consideran que la vida les va bien o muy bien, cerca de una cuarta parte considera que les va regular y sólo un pequeño grupo afirma que le va mal $(3,5 \%)$, lo que muestra en general una elevada satisfacción con el funcionamiento de su vida. Teniendo en cuenta que el grado de satisfacción existencial de los jóvenes españoles se sitúa en torno al $85 \%$ (Injuve, 2004) y dado el cúmulo de problemas y dificultades añadidas a que debe enfrentarse la población de jóvenes en dificultad social, cabe hablar de una satisfacción muy notoria, aunque, por supuesto, no suficiente.

Profundizando en este contraste entre los jóvenes en dificultad social y los datos globales para la población juvenil española, vemos cómo los primeros se muestran razonablemente satisfechos con la marcha de sus vidas. Están mucho más preocupados 
con todo lo relacionado con el mundo del trabajo y la ocupación profesional y con la situación económica y los bienes materiales, y muy mediatizados -en positivo y en negativo- por su situación personal y, especialmente, por su capacidad de mantener una vida independiente y por su estabilidad emocional. Aunque preocupados por las relaciones personales, familiares, de amistad y de pareja, lo están mucho menos que la media de los jóvenes españoles, y no sienten especial atracción por las diversiones y los viajes. Estas diferencias, en muchos casos notables, entre ambas poblaciones juveniles, nos ofrecen unas claves muy determinadas para interpretar la realidad y orientar la intervención socioeducativa.

\section{Los efectos de la intervención socioeducativa}

El tercer objetivo de la investigación plantea describir, analizar y valorar la incidencia del modelo de intervención socioeducativa utilizado en el tránsito de los adolescentes y jóvenes en dificultad social a una vida adulta independiente y responsable. Desde el análisis de los recuerdos y percepciones de los jóvenes sobre los programas de empleo, observamos cómo prácticamente todos los jóvenes entrevistados piensan que es importante que existan recursos como estos programas de empleo, dirigidos tanto a mayores de edad (100\%) como a menores de edad (96,5\%).

En cuanto a los aspectos que los jóvenes recuerdan del programa de empleo, habría que destacar:

- En primer lugar, recuerdan el programa como el espacio en el que se les ayudó a buscar trabajo, se les enseñó lo necesario para ello, se les puso en contacto con sus primeros empleos y se les apoyó a la hora de mantenerlos.

- El segundo recuerdo más fuerte y consistente es la relación personal establecida con los educadores y el buen trato personal recibido; la ayuda prestada y el interés personal que sintieron hacia sí mismos, hacia sus problemas, expectativas y posibilidades de futuro.

- En tercer lugar, hacen referencia al recuerdo de un buen ambiente, de compañerismo; un ambiente agradable y respetuoso hacia ellos y hacia el grupo de jóvenes que acudían al programa.

- En cuanto a los aspectos negativos, los jóvenes recuerdan:

- Situaciones de tensión o mal ambiente, que atribuyen esencialmente a las relaciones entre iguales, y, en concreto, a la presencia de ciertas personas o grupos que mediatizaban la relación y la hacían poco atractiva o desagradable $(21 \%)$.
- Otros recuerdos poco gratos son la dificultad para encontrar trabajo, la impaciencia en la espera hasta encontrar el trabajo adecuado, junto a la opinión de que algunos trabajos eran malos, poco gratificantes, o de baja calidad. También se menciona (entre el $4 \%$ y el $9 \%$ de la muestra lo hace) la lejanía de los centros y la necesidad de desplazarse para acceder a ellos.

Los jóvenes se sintieron especialmente atendidos y apoyados desde los programas (puntuaciones de 9 y 8,6 sobre 10 , con una baja desviación típica), y valoran de forma muy elevada y homogénea la cualificación de los educadores (media de 8,97, desviación típica de 1,37 ), relacionando directa e intensamente su recuerdo de los educadores con los aprendizajes relativos a la autonomía, la independencia y la maduración personal.

En cuanto a su paso por el sistema de protección de menores, un grupo amplio de jóvenes (31,9\%) tiene un buen recuerdo de los educadores y de los centros de menores. Los aspectos que los jóvenes valoran mejor de su estancia en protección de menores son el ambiente existente, las buenas relaciones con sus compañeros, el apoyo y el buen trato recibidos, y su relación con los educadores/as de los centros.

Un grupo de jóvenes muestra, sin embargo, un recuerdo negativo $(20,2 \%)$ o ambivalente $(10,9 \%)$ sobre su estancia en estos dispositivos. Como el reflejo de un espejo, lo que menos gustó a estos jóvenes durante su estancia en el sistema de protección fueron también los compañeros y la convivencia, y la relación con educadores/as, aunque en proporciones más reducidas. Éstos son, junto a la percepción de la normativa de los centros como restrictiva y excesiva, los elementos más relevantes de sus respuestas.

Cuando se preguntó a los jóvenes sobre los aprendizajes obtenidos durante su estancia en protección de menores, dos tercios de ellos opinaron que les sirvió para madurar, avanzar hacia la autonomía y la independencia, y para aprender a convivir y a relacionarse. Poder estudiar y aprender es otro de los beneficios declarados, junto a la entrada en el mundo del trabajo. Además, los aprendizajes obtenidos por los jóvenes durante su estancia en protección de menores presentan una relación estrecha y directa con la percepción del joven de que consigue los recursos suficientes para cubrir sus necesidades personales.

En sus propias palabras, lo que aprendieron a través de la intervención socioeducativa fue:

A no dar todo por perdido... Me han sido útiles muchas cosas, aprender bastante de la nueva vida que te espera... A pensar a fondo lo que realmente quiere... El compañerismo, el respeto y la 
paciencia... A saber las cosas que hace la gente normal... Aguantar lo que te digan... A no valorar a ninguna persona por su apariencia.

Encontramos que los jóvenes no coinciden en su opinión global sobre el sistema de protección de menores y sobre el programa de empleo. Los jóvenes que no valoran especialmente su estancia en protección de menores, o que la ven como algo perjudicial, sí han encontrado, sin embargo, en el programa de empleo alicientes importantes. Y viceversa, los jóvenes que se encuentran a disgusto y muy a disgusto en el programa de empleo, se encontraban a gusto en protección de menores.

\section{El rol de los profesionales intervinientes}

Con el cuarto objetivo, la investigación pretende determinar el perfil, los principios de actuación, los planteamientos, las estrategias y las expectativas de los profesionales implicados, así como sus aportaciones al modelo de intervención socioeducativa con adolescentes y jóvenes en dificultad social. La percepción de la realidad que tienen los profesionales nos puede ayudar, sin duda, a conocer sus expectativas y motivaciones en relación al trabajo que realizan, a lo que realimenta su intervención y a lo creen que proporciona determinados escenarios de tránsito a la vida adulta a la población con la que trabajan.

Así, entre las características personales que más facilitan el trabajo con los jóvenes en dificultad social, los profesionales mencionan, en este orden:

- La empatía, la sensibilidad, la proximidad y la cercanía a los jóvenes.

- Las habilidades sociales y de comunicación.

- Las actitudes de flexibilidad, tolerancia y respeto hacia los jóvenes.

Se aprecia un acuerdo importante, sólido y cohesionado entre los profesionales a la hora de hacer esta valoración, que hace referencia a cualidades proactivas de comprensión y apoyo. Quedan claramente fuera de sus respuestas los planteamientos más restrictivos, como los referidos al control o la disciplina.

Junto a la importancia de tener experiencia previa en intervención socioeducativa con adolescentes o jóvenes en dificultad social, y en orientación profesional/laboral, los profesionales consideran aspectos básicos en su formación, por este orden:

- La metodología de intervención y, de forma más concreta, las técnicas de comunicación, habilidades sociales y técnicas de resolución de conflictos.
- El conocimiento de la población con la que se está trabajando, su contexto y sus peculiaridades personales, sociales y educativas.

- Los estudios reglados que doten de un marco teórico en ciencias sociales, y de forma más concreta en Educación, Psicología y Sociología.

En este sentido, los profesionales están de acuerdo en que la elección de uno u otro marco teórico incidirá de forma directa y significativa en la eficacia de la intervención socioeducativa desarrollada (80\%).

El modelo sistémico-complejo-ecológico y el cognitivo-conductual son los que mayor adhesión concentran. Los profesionales consideran, por otra parte, que su formación no se lleva a cabo con la necesaria frecuencia y sistematicidad, lo que, en su opinión, supone una grave limitación, máxime cuando se reconoce que el educador, con su bagaje personal, su experiencia y formación, es la principal herramienta de trabajo, muchas veces la única, en este ámbito.

Los profesionales plantean cómo ellos mismos disponen de pocos recursos, estrategias y flexibilidad para abordar situaciones imprevistas o inciertas, una parte importante de lo que pueden encontrar cada día. La flexibilidad en el diseño de itinerarios está, además, directa y significativamente relacionada con el interés por la participación de los jóvenes en la toma de decisiones sobre su futuro personal, social y laboral, junto a los educadores y otros profesionales que trabajan con ellos $\left(r^{2}=0,456\right.$, $p \leq 0,000 ; r$ de Pearson $\geq 0,500$ ).

Los profesionales consideran, por otra parte, muy bajo el nivel de participación y de colaboración de las familias de los jóvenes en dificultad social en las actuaciones planteadas para facilitar su tránsito a la vida adulta. Es significativo, en este sentido, que estos programas tampoco están orientados, ni dedican espacios importantes de trabajo, a la intervención con familias, que se reconoce como uno de sus puntos débiles, y que se relaciona directamente con esta percepción de los profesionales.

Interrogados sobre aquellos indicadores que nos pueden aproximar a un mejor conocimiento de los resultados de la intervención socioeducativa en este ámbito, los profesionales mencionan los siguientes, por este orden:

- La estabilidad laboral de los jóvenes en dificultad social.

- La estabilidad emocional y el proyecto de vida de los jóvenes.

- Una vivienda estable y digna.

Como elementos predictores del fracaso en el tránsito a la vida adulta, se mencionan los hábitos deficitarios en cuanto a la autonomía personal, la incapa- 
cidad para mantener el empleo, las relaciones sociofamiliares conflictivas y la presencia de redes sociales manipuladas. En cuanto a la intervención socioeducativa, los profesionales consideran mayoritariamente que ésta es eficaz, y que favorece el tránsito a una vida adulta independiente y responsable de los jóvenes en dificultad social (puntuación de 8,5 sobre 10 , desviación típica de 1,699 ). Coinciden en que, cuando finaliza la intervención socioeducativa, los jóvenes en dificultad social tienen los conocimientos necesarios para la búsqueda activa de empleo, aunque en general no han adquirido un nivel adecuado de capacitación para mantener el puesto de trabajo y adaptarse a los requerimientos de éste. Están también bastante de acuerdo en apreciar que la intervención socioeducativa produce cambios significativos en el comportamiento social responsable, y en la adquisición y consolidación de unos valores éticos más firmes. Sin embargo, en torno a la tercera parte de ellos opina que los jóvenes en dificultad social o bien no transitarán de forma adecuada a la vida adulta, o bien sucumbirán a los riesgos de la exclusión social a los pocos años de abandonar los programas que los atienden.

Los aspectos de la intervención socioeducativa que los profesionales consideran más eficaces son, por orden de prioridad:

- Fomentar habilidades de autonomía.

- Dar protagonismo a los adolescentes y los jóvenes.

- Cuidar la relación entre los educadores y los jóvenes.

- Realizar una buena planificación y coordinación de actuaciones.

- El propio trabajo del educador y su formación.

Y los obstáculos, impedimentos o limitaciones más frecuentes en este tipo de intervención socioeducativa son, en este orden:

- La falta de apoyos y recursos institucionales.

- La descoordinación entre los recursos existentes.

- La falta de motivación del joven.

- Un entorno sociofamiliar que obstaculiza la intervención.

Para los profesionales, por otra parte, un elemento que mejora la eficacia de la intervención es el trabajo coordinado e interdisciplinar. Consideran además que los recursos económicos destinados a este tipo de intervención socioeducativa (inversiones en salarios, infraestructuras, materiales) y a favorecer el tránsito a la vida adulta de los jóvenes en dificultad social son insuficientes, y tampoco consideran adecuados los recursos destinados específicamente a los jóvenes inmigrantes. Se defiende con claridad una propuesta que ya recogen investigaciones anteriores en este ámbito: la Administración debe mantener los recursos de intervención socioeducativa para facilitar el tránsito a la vida adulta de los jóvenes en dificultad social después de que estos hayan cumplido la mayoría de edad. Ésta es una conclusión respaldada por la mayoría de los profesionales, y con una valoración muy elevada (valoración de 9,14 sobre 10 ).

\section{El papel de los empresarios}

Un último objetivo del estudio plantea conocer la incidencia y la valoración de los programas de inserción sociolaboral en el ámbito empresarial: sus debilidades, fortalezas y posibilidades. A este respecto, prácticamente las dos terceras partes de los empresarios consultados consideran que estos programas funcionan bien, aunque un tercio piensa que podrían mejorar. En cualquier caso, el 98,9\% de los empresarios continuaría contratando jóvenes a través de los programas de empleo.

En general, los empresarios consideran que lo mejor de los programas de inserción sociolaboral es el apoyo y la ayuda que prestan a los jóvenes, y ponen de relieve su interés por el carácter social del ingreso de los jóvenes en el mundo del trabajo, y su visión solidaria de la labor de los educadores y la intervención socioeducativa con jóvenes en dificultad social. En segundo lugar, reflejan otro elemento relevante, esta vez para ellos y su empresa, como que facilitan la contratación y ayudan al empresario a seleccionar a sus trabajadores. Un tercer elemento especialmente valorado es la buena atención recibida desde los programas y sus profesionales, lo que muestra un buen clima de trabajo entre ambos y, sin duda, tiene sus repercusiones en la facilitación del empleo a los jóvenes avalados por los programas y en la continuidad en él.

\section{En cualquier caso, el 98,9\% de los empresarios continuaría contratando jóvenes a través de los programas de empleo.}

En cuanto a los planteamientos de mejora que hacen los empresarios, en primer lugar mencionan la necesidad de establecer un mayor control de los programas en la selección inicial de candidatos para el empleo, y de buscar un mayor ajuste entre las características de aquéllos y las necesidades de la empresa. El segundo grupo de propuestas de mejora no es tal, sino que más bien hace referencia a una 'queja' 
sobre las actitudes y los comportamientos de algunos jóvenes en el trabajo, aunque la percepción de los empresarios sobre la creación de situaciones conflictivas por parte de los jóvenes es bastante baja (3,8 puntos sobre 10), y con una gran dispersión en la respuesta (desviación típica $=3,07$ ). Esto apunta a una tónica general de ausencia de conflicto, aunque con la presencia de casos aislados 0 situaciones puntuales de intensa conflictividad.

La búsqueda de buenos resultados económicos en el rendimiento de trabajo no es el único elemento que hace que los empresarios contraten a estos jóvenes, sobre todo teniendo en cuenta que la valoración media que los empresarios dan a los usuarios de los programas que trabajan para ellos no pasa del aprobado (5,95 puntos sobre 10). Los empresarios consideran, de forma mayoritaria, que la contratación de jóvenes supone una 'inversión de futuro', y no tanto un ahorro económico -aunque también-, o una mejora sobre la contratación de personas de más edad. Hay que tener en cuenta que los jóvenes son una buena opción para los empresarios: por término general, no demandan salarios elevados, aunque tampoco aceptan cualquier tipo de trabajo.

En este sentido, los empresarios manifiestan mayoritariamente que las empresas pueden ayudar a los jóvenes con problemas sociales, proporcionándoles un empleo que favorezca su socialización y les introduzca en el mundo laboral, enseñándoles un oficio, apoyándoles y dándoles una oportunidad para integrarse laboralmente, haciendo que se sientan integrados socialmente y ayudándoles a entender que tienen algo que aportar a la sociedad. Sin embargo, los mismos empresarios se muestran poco dispuestos a contratar jóvenes que estén sometidos, o lo hayan estado, a medidas judiciales, que hayan tenido problemas de consumo de sustancias tóxicas, que sean menores de edad, que tengan alguna discapacidad o sean de otras etnias, y muestran reticencias a contratar a mujeres con hijos, estudiantes y mujeres en general.

\section{Algunas aplicaciones de los resultados obtenidos: diez propuestas para construir una sociedad sostenible}

Acompañar a los adolescentes y jóvenes con mayores dificultades para transitar a una vida adulta independiente y responsable es una tarea compleja, sensible y, en ocasiones, dura. Tener la oportunidad y el valor de intervenir en un momento crucial de la vida de las personas, en un escenario vital para la sociedad y para su futuro, comporta una gran responsabilidad, una carga de riesgo y la necesidad de comprobar, al cabo del tiempo, que el trabajo realizado sirvió para cambiar la realidad, para aproximarnos a una sociedad más justa, equilibrada, sostenible. Investigar acerca de todo ello es parte de esa necesidad, la necesidad de conocer, de saber, de contemplar en la distancia para qué sirvió ese gran esfuerzo social y personal, desde la idea de que la investigación es parte y alimento de una acción realmente transformadora.

Seguidamente, y de forma sintética, reflejamos algunas propuestas elaboradas conjuntamente por el equipo de investigación, y los educadores y profesionales intervinientes en este tipo de situaciones:

1. Potenciar y priorizar los programas de inserción sociolaboral para mayores y menores de edad, como punto nodal del sistema y foco del esfuerzo que se desarrolla en nuestra sociedad para integrar a la población de jóvenes en dificultad social, y convertirla en colectividad adulta independiente y responsable, ayudando así a construir de forma sostenible y equilibrada la sociedad del futuro.

2. Ampliar el espacio de intervención con mujeres adolescentes y jóvenes en dificultad social, y mantener la intervención con jóvenes inmigrantes, desde la discriminación positiva y la incorporación en igualdad de condiciones a la cultura del trabajo.

3. Incrementar la proporción de empresas 'activas' en estos programas, potenciando el sentido realmente solidario de las empresas colaboradoras y trabajando en la línea más social de la responsabilidad social de empresa.

4. Abordar de forma sistemática y continuada el trabajo socioeducativo con las familias de los jóvenes en dificultad social, definida como una 'tarea pendiente' por los propios profesionales.

5. Considerar como criterios, en los procesos de selección de personal y de formación de profesionales, los recomendados y propuestos por los propios profesionales: la empatía, la sensibilidad, la proximidad y la cercanía al joven, las habilidades sociales y de comunicación, la actitud de flexibilidad, la tolerancia y el respeto hacia los jóvenes, y la experiencia previa en intervención socioeducativa con adolescentes y jóvenes en dificultad social, y en orientación profesional y laboral.

6. Potenciar la flexibilidad en la intervención y la formación, para abordar situaciones de incertidumbre; revisar críticamente y afianzar el papel de los diferentes elementos del modelo de estrategias flexibles de intervención socioeducativa.

7. Incrementar la participación de los jóvenes en las actividades y la organización del programa, para avanzar en la toma de decisiones personalizada y en la mejora de las relaciones entre iguales.

8. Gestionar mayores apoyos institucionales -vivienda, empleo, becas de formación- para 
este grupo poblacional, como contrapartida a la dura vivencia de tener que 'salir adelante solos/as' y de la falta de apoyos externos que, hasta ahora, les ha acompañado.

9. Incrementar la coordinación interinstitucional, especialmente entre programas de empleo y sistemas de protección, y trabajar conjuntamente los aspectos deficitarios observados.

10. Avanzar en la realización de nuevas investigaciones, contrastando diferentes modelos de inter-

\section{Bibliografía}

(2006): PISA 2006. Programa para la Evaluación Internacional de Alumnos de la OCDE. Informe español, Madrid, Ministerio de Educación y Ciencia [rhttp://www.mec.es/multimedia/00005713. pdf>].

BENDIT, R.; y STOKES, D. (2004): “Jóvenes en situación de desventaja social: políticas de transición entre la construcción social y las necesidades de una juventud vulnerable", Revista de Estudios de Juventud, $\mathrm{n}$ 으, 5 , págs. 115-131.

CACHÓN, L. (2004): Las políticas de transición: estrategia de actores y políticas de empleo juvenil en Europa, Madrid, Instituto de la Juventud.

- (1998): Políticas de inserción de los jóvenes en los mercados de trabajo en la Unión Europea, Montevideo, Cinterfor; Organización Internacional del Trabajo.

CASAS, F.; y MONSERRAT, C. (2009): "Sistema educativo e igualdad de oportunidades entre los jóvenes tutelados: estudios recientes en el Reino Unido", Psicothema. vol. 21, no 4, págs. 543-547 [disponible en: 〈http://www.psicothema.com/pdf/3668.pdf〉].

COMISIÓN DE LAS COMUNIDADES EUROPEAS (2003): Comunicación de la Comisión al Consejo, al Parlamento Europeo, al Comité Económico y Social Europeo y al Comité de las Regiones. Informe conjunto sobre la inclusión social en el que se resumen los resultados del examen de los planes nacionales de acción en favor de la inclusión social (2003-2005), Bruselas, vención socioeducativa y sus resultados en el tránsito de los jóvenes en dificultad social a la vida adulta.

Con estas propuestas, terminamos este artículo, no sin antes agradecer a todos los que han estado implicados en la investigación su esfuerzo y su interés, que es el nuestro, por avanzar hacia una sociedad más justa socialmente, más solidaria y más equilibrada ambientalmente, una sociedad que sea realmente sostenible.

- (2001): Un nuevo impulso para la juventud europea, Bruselas, Comisión de las Comunidades Europeas.

CONDE GUTIÉRREZ DEL ÁLAMO, F. (2005): “Los adolescentes ante un futuro laboral que cambió", en Actas del Congreso 'Ser adolescente, hoy', Madrid, Fundación de Ayuda contra la Drogadicción.

DU BOIS-REYMOND, M.; y LÓPEZ BLASCO, A. (2004): “Transiciones tipo yo-yo y trayectorias fallidas: hacia las políticas integradas de transición para los jóvenes europeos", Revista de Estudios de Juventud, no 65, págs. 11-29.

DU BOIS-REYMOND et al. (2002): How to avoid cooling out? Experiences of young people in their transitions to work across Europe, Youth Policy and Participation Research Project [<http://www.iris-egris.de/yoyo/pdf/Yoyowor kingpaper2.pdf`].

FERNÁNDEZ DEL VALLE, J. (1998): Y después... ¿qué? Estudio de casos que fueron acogidos en residencias de protección de menores en el Principado de Asturias, Oviedo, Consejería de Servicios Sociales del Principado de Asturias.

GARCÍA BARRIOCANAL, C.; IMAÑA, A.; y DE LA HERRÁN, A. (2007): El acogimiento residencial como medida de protección al menor, Madrid, Defensor del Menor de la Comunidad de Madrid. 
GONZÁLEZ-ANLEO, J.; y GONZÁLEZ BLASCO, P. (dirs.): Jóvenes españoles 2010, Madrid, Fundación SM.

GOYETTE, M. et al. (2007): “Le soutien au passage à la vie adulte des jeunes recevant des services des centres jeunesse", Éducation et Francophonie. Revue Scientifique Virtuelle, vol. 35, $\mathrm{n}-1$.

GOYETTE, M.; y TURCOTTE, D. (2004): "La transition vers la vie adulte des jeunes qui ont vécu un placement: un défi pour les organismes de protection de la jeunesse", Revue Service Social, nํ51, págs. 29-44.

INGLÉS, A. et al. (2005): Aprendiendo a volar. Estudio para el análisis de los programas europeos Mentor 15 y Ulises, dedicados al proceso de socialización de adolescentes y jóvenes tutelados que han alcanzado la mayoría de edad o están cerca de ella, Murcia, Fundación Diagrama.

INSTITUTE FOR REGIONAL INNOVATION AND SOCIAL RESEARCH (2005): Thematic Study on Policy Measures Concerning Disadvantaged Youth, Tubinga, Institute for Regional Innovation and Social Research.

INSTITUTO DE LA JUVENTUD (2004): Informe Juventud de España 2004, Madrid, Instituto de la Juventud.

JIMÉNEZ BARCA, A. (2005): "La generación de los mil euros”, El País, 23 de octubre, págs. 23-25.

LENZ-RASHID, S. (2006): “Employment experiences of homeless young adults: Are they different for youth with history of foster care?", Children and Youth Services Review, vol. 28, nํㅜ 3, págs. 235-269.

LERBET-SERENI, F. (2004): La relation pédagogique: éclairage systémique et travail des paradoxes, Montpellier, Réseau Intelligence de la Complexité [khttp://www.intelligence-complexite.org/ fileadmin/docs/ateliers/6_doc1.pdfs].

LEPOUTRE, D. (1999): “La culture de rue adolescente dans les grands ensembles de banlieue", en MORIN, E. (1999): Le défi du XXIe siècle. Relier les connaissances, París, Éditions du Seuil, págs. 359-363.

LOMAS, M. (2005): Estudio comparativo del acogimiento residencial de la Comunidad de Madrid en el contexto nacional, Madrid, Opción 3 [<http://www.institutoopcion3.es/Archivos/Est udio\%20Acogimiento\%20Residencial.pdf $>$ ].

LÓPEZ BLASCO, A. (2007): “Reacciones de la Unión Europea a la situación de los/las jóvenes: planes de empleo, estrategia Lisboa, políticas integrales de reforma", Revista de Estudios de Juventud, no 77 , págs. 13-29.

MACHADO PAÍS, J.; y POHL, A. (2004): "Los dilemas del reconocimiento del aprendizaje informal", en
Revista de Estudios de Juventud, $\mathrm{n} \div 05$, págs. 83-98.

MELENDRO, M. (dir.) [2010]: El tránsito a la vida adulta de los jóvenes en dificultad social, Madrid, Universidad Nacional de Educación a Distancia.

- (dir.) [2007]: Estrategias educativas con adolescentes y jóvenes en dificultad social. El tránsito a la vida adulta en una sociedad sostenible, Madrid, Universidad Nacional de Educación a Distancia.

MINISTERIO DE EDUCACIÓN, POLÍTICA SOCIAL Y DEPORTE (2008): Datos y cifras. Curso escolar 20082009, Madrid, Ministerio de Educación, Política Social y Deporte.

MORIN, E. (2005): Introducción al pensamiento complejo, Barcelona, Gedisa.

NÚÑEZ, V. (1990): Modelos de educación social en la época contemporánea, Barcelona, PPU.

PROYECTO TELÉMACO (2005): El presente: una apuesta de futuro. Buenas prácticas en la atención a jóvenes en proceso de inserción sociolaboral y autonomía, Murcia, Fundación Diagrama.

RAHONA, M.; y VAQUERO, A. (2003): "La transición de los jóvenes desde el sistema educativo al mercado de trabajo. Una aproximación para la Comunidad de Madrid", en Actas de las V Jornadas de Economía Laboral. 9-11 julio, Reus, Universitat Rovira i Virgili.

REILLY, T. (2003): "Transition form care: Status and outcomes of youth who age out of foster care", Child Welfare, $\mathrm{n}^{0} \mathrm{82}$, págs. 727-746.

STEIN, M. (2006): "Research review: Young people leaving care", Child and Family Social Work, vol. 11, no 3, págs. 273-279.

TARÍN, M.; y NAVARRO, J. (2006): Adolescentes en riesgo. Casos prácticos y estrategias de intervención socioeducativa, Madrid, CCS.

VÉLAZ DE MEDRANO, C. (2004): Intervención educativa y orientadora para la inclusión social de menores en riesgo. Factores escolares y socioculturales, Madrid, Universidad Nacional de Educación a Distancia.

WALTHER, A.; y POHL, A. (2007): “Jóvenes desfavorecidos en Europa. Constelaciones y respuestas políticas", Revista de Estudios de Juventud, ํㅜ 77, págs. 155-172.

YERGUEAU, E.; PAUZÉ, R.; y TOUPIN, J. (2007): “L'insertion professionnelle et l'adaptation psychosociale des jeunes adultes ayant reçu des services des centres jeunesse", Intervention, nํㅜ 127 , págs. 58-69. 